International Journal of Engineering \& Technology, $7(3.29)(2018) 269-271$
SPC
International Journal of Engineering \& Technology
Website $: \frac{w w . s c i e n c e p u b c o . c o m / i n d e x . p h p / I J E T}{2}$
Research paper

\title{
Dual tree complex wavelet transform based image denoising for Kalpana satellite images
}

\author{
Naga Lingamaiah Kurva ${ }^{1}$, S. Varadarajan ${ }^{1}$ \\ ${ }^{1}$ Department of E. C. E, S. V. University College of Engineering \\ *Corresponding author E-mail: nagalingamaiahk@gmail.com
}

\begin{abstract}
This paper presents a new algorithm to reduce the noise from Kalpana Satellite Images using Dual Tree Complex Wavelet Transform technique. Satellite Images are not simple photographs; they are pictorial representation of measured data. Interpretation of noisy raw data leads to wrong estimation of geophysical parameters such as precipitation, cloud information etc., hence there is a need to improve the raw data by reducing the noise for better analysis. The satellite images are normally affected by various noises. This paper mainly concentrates on reducing the Gaussian noise, Poisson noise and Salt \& Pepper noise. Finally the performance of the DTCWT wavelet measures in terms of Peak Signal to Noise Ratio and Structural Similarity Index for both noisy \& denoised Kalpana images.
\end{abstract}

Keywords: DTCWT; Gaussian Noise; Noise Reduction; Poisson Noise; PSNR; Salt \& Pepper Noise.

\section{Introduction}

A lot of research work has been concentrated towards noise reduction techniques in digital image processing. Mainly the noise is introduced in image during either acquisition or transmission of the images; there are various algorithms \& techniques to study, estimate and to reduce the noise from images. Applying image processing techniques to Satellite images are not just like applying techniques to test images such as lenna, cameraman, mandrill etc.,. The satellite images are not just simple photographs, but contain various bands of measured data. Reducing noise from satellite image is a challenge for the researcher in digital image processing.

This paper concentrates mainly on Kalpana Satellite Data which is taken from Indian Meteorological Department. Kalpana Satellite is the first Indian meteorological Satellite built by Indian Space Research Organization (ISRO). The Satellite comprises two payloads they are Very High Resolution Radio Meter (VHRR) and Data Relay Transponder (DRT) for collection and transmission of meteorological, hydrological and oceanographic data. The VHRR sensor operates in three wavelength bands which are Visible Band (VIS), Water vapor band (WV) and Thermal Infrared band (TIR). Here, image processing algorithms and denoising techniques are applied to the TIR band channel images.

\section{Related work}

Image noise is nothing but undesired changes of brightness information or color information in images. There changes may occur mainly during image acquisition or transmission. This paper mainly concentrates on Gaussian noise, Poisson noise, and Salt \& Pepper noises. The important characteristic of these noises is there is no relationship between noisy and other pixels. The black and white pixels introduced in the image called as Salt \& Pepper noise. The Poisson noise is mostly occurred in lighter parts of an image.
The image denoising techniques are ever-growing in digital image processing. There are number of spatial domain filters to reduce noise in images. Different filters are best suitable for different noises. For example, Median filter is a spatial filter which is efficient to reduce Salt \& Pepper noise in an image. In image processing mostly Median filter \& Wiener filters are used to reduce various noises. If the size of mask varies, the efficiency of the filters also varies. As the mask size increases from $3 \times 3$ to $7 \times 7$ the efficiency of Median filter reduces whereas the efficiency of Wiener filter increases.

The spatial filters are well suitable for test images but not recommended for satellite images. The performance of spatial filters has drastic change when applied to satellite images. For example, if the satellite image consists of cloud information, the edges of cloud consider as Salt noise. Similarly various parameters are degradable by spatial filters.

Wavelet filters are introduced to get better results in digital image processing, which are also suitable for satellite images. This paper shows the performance of some basic wavelet filters in reduction of noise from KALPANA satellite image. Here Daubechies wavelet, Bi-orthogonal wavelet, Symlet and Coiflet's are used to reduce the noise. Finally, the satellite noisy images are denoised by using Dual Tree Complex Wavelet Transform (DTCWT) which gives better results.

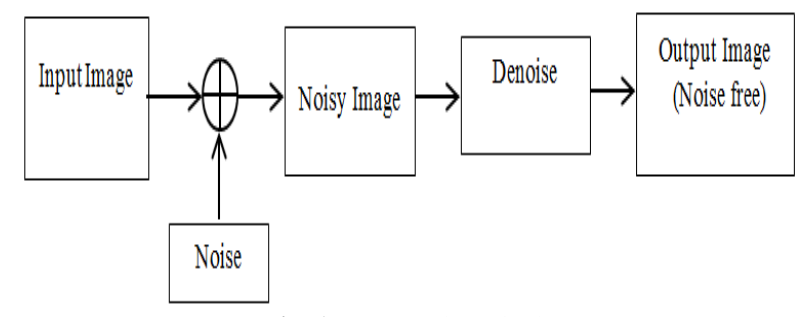

Fig. 1: Proposed Method. 
In general, the noisy images are directly applied to denoising algorithms to reduce the noise. Here one may not know whether the algorithm designed reduces the noise or removes the other important information from the image. Fig. 1 shows the proposed method where the noise is added intentionally to the satellite image, then applies to the algorithm designed to reduce the noise, and compare the denoised image with the original image.

\section{Dual tree complex wavelet transform}

The Dual Tree Complex Wavelet Transform is the advanced to the basic Discrete Wavelet transform. DTCWT is advantageous compared to DWT i.e., the DTCWT possessing additional properties which are very important in Digital image processing. Dual Tree Complex Wavelet Transform is nearly Shift invariant and directionally selective in two and higher dimensions which gives the better performance compared to other wavelet transforms. When applying the wavelet transforms to Satellite Images, it is very important to be shift invariant and directionality. Otherwise, the information provided by the satellite image will be lost to some extent which gives undesired results.

The DTCWT can be implemented using two distinct sets of separable $2 \mathrm{D}$ wavelet bases.

$\psi 1,1(\mathrm{x}, \mathrm{y})=\varphi \mathrm{h}(\mathrm{x}) \psi \mathrm{h}(\mathrm{y}), \psi 2,1(\mathrm{x}, \mathrm{y})=\varphi \mathrm{g}(\mathrm{x}) \psi \mathrm{g}(\mathrm{y}), \psi 3,1(\mathrm{x}$, $y)=\varphi g(x) \psi h(y), \psi 4,1(x, y)=\varphi h(x) \psi g(y)$,

$\psi 1,2(\mathrm{x}, \mathrm{y})=\psi \mathrm{h}(\mathrm{x}) \varphi \mathrm{h}(\mathrm{y}), \psi 2,2(\mathrm{x}, \mathrm{y})=\psi \mathrm{g}(\mathrm{x}) \varphi \mathrm{g}(\mathrm{y}), \psi 3,2(\mathrm{x}$, $y)=\psi g(x) \varphi h(y), \psi 4,2(x, y)=\psi h(x) \varphi g(y)$,

$\psi 1,3(\mathrm{x}, \mathrm{y})=\psi \mathrm{h}(\mathrm{x}) \psi \mathrm{h}(\mathrm{y}) \psi 2,3(\mathrm{x}, \mathrm{y})=\psi \mathrm{g}(\mathrm{x}) \psi \mathrm{g}(\mathrm{y}), \psi 3,3(\mathrm{x}$, y) $=\psi g(x) \psi h(y) \psi 4,3(x, y)=\psi h(x) \psi g(y)$

The relationship between wavelet filters $\mathrm{h}$ and $\mathrm{g}$ is shown below

$\mathrm{g} 0(\mathrm{n}) \approx \mathrm{h} 0(\mathrm{n}-1)$, for $\mathrm{j}=1 \quad \mathrm{~g} 0(\mathrm{n}) \approx \mathrm{h} 0(\mathrm{n}-0.5)$, for $\mathrm{j}>1$

First, convert the RGB input image into $\mathrm{YCbCr}$ (luminance and Chrominance) components. As most of the information available in luminance component, luma channel of the image is used to process. Chrominance components preserve the edge information which can be added in the last step. The process of proposed algorithm is shown in Fig.2.

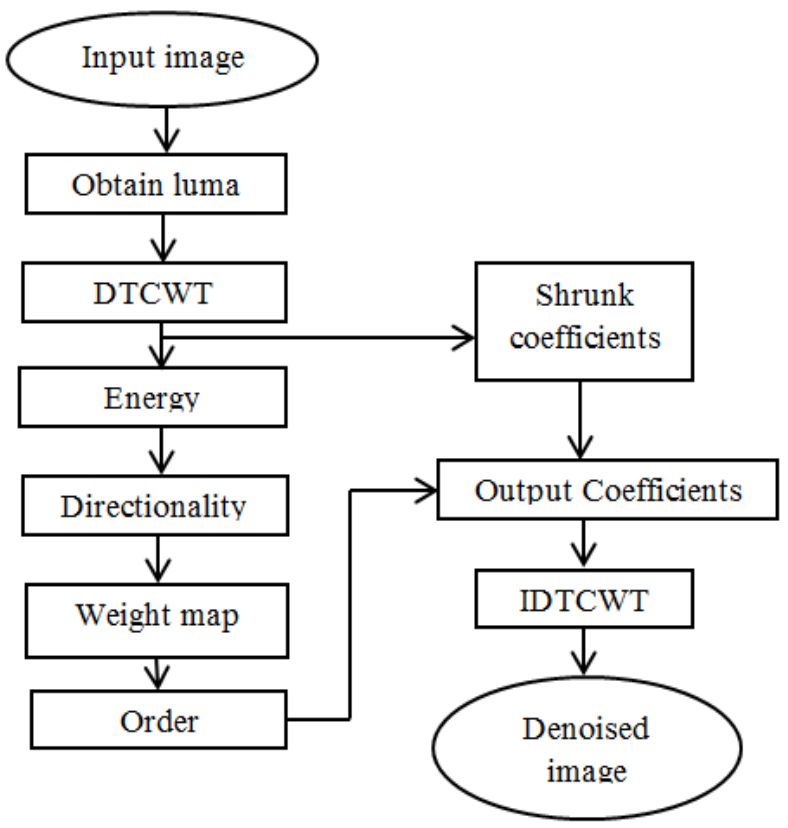

Fig. 2: Flowchart of the Implementing Method.

\section{Results and discussions}

The algorithms developed to reduce noise are implemented using MATLAB. The performance of wavelet filters and Dual Tree Complex Wavelet Transform is tested on still images and KALPANA satellite images. The performance metrics Peak Signal to Noise Ratio (PSNR) \& Structural Similarity module measures the efficiency of noise reduction algorithms.

Peak Signal to Noise Ratio $=10 \log _{10}\left[\frac{(255)^{2}}{\mathrm{MSE}}\right]$

Mean Square Error $(M S E)=\frac{1}{n \times m} \sum_{i=1}^{n} \sum_{j=1}^{m}\left(x_{i, j}-y_{i, j}\right)^{2}$

\subsection{Original \& noisy images}

(A)

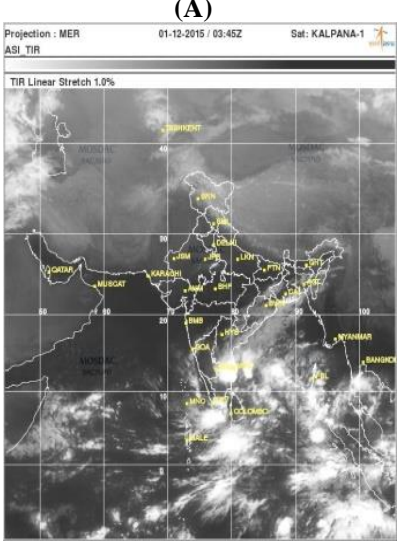

(C)

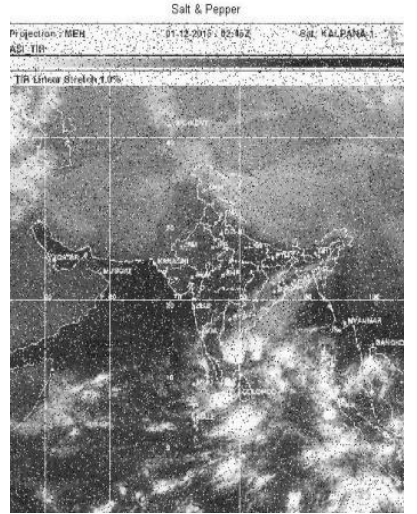

(B)

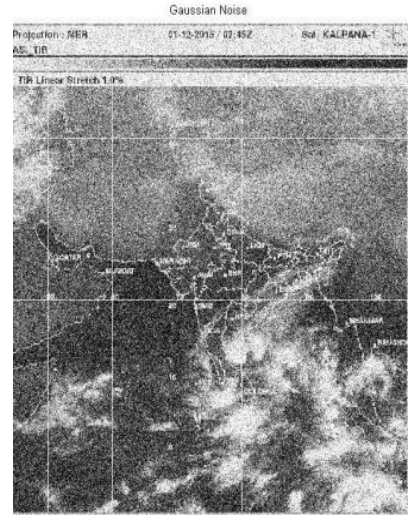

(D)

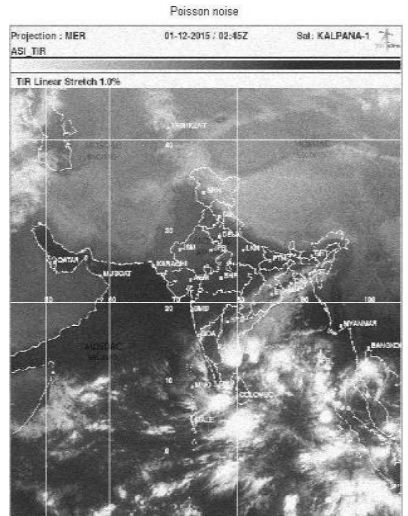

Fig. 3: A) Original Kalpana Image, B) Gaussian Noisy Image, C) Salt \& Pepper Noisy Image, D) Poisson Noisy Image.

Fig. 3 shows the original Kalpana image and noisy images which are used as input images for denoising algorithms to reduce the noise. Gaussian noise is distributed over the image with minimal variations, Salt \& Pepper noise is clearly visible as white and dark points on the image, and Poisson noise concentrated mainly on brighter areas of the image.

\subsection{Denoising using wavelet filters}


(A)

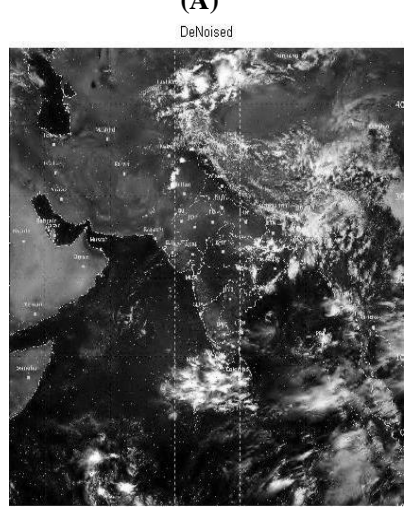

(C)

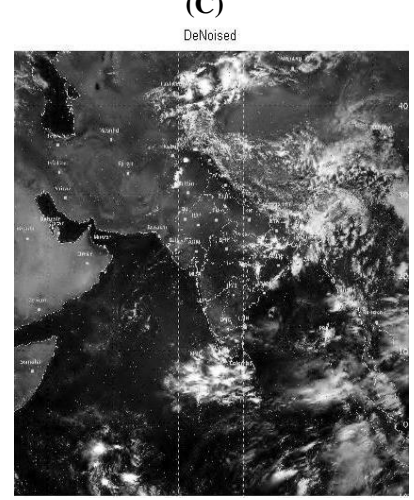

(B)

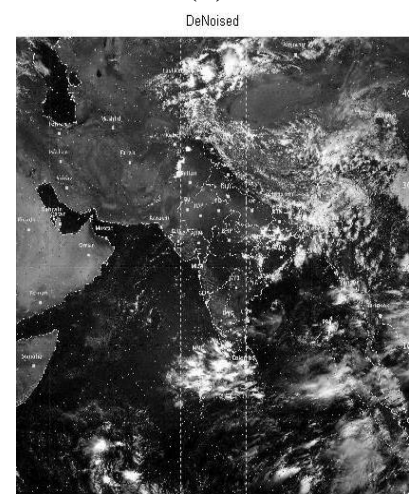

(D)

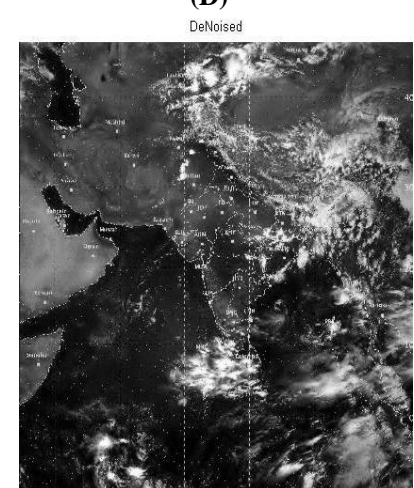

Fig. 4: Denoised Images for Salt \& Pepper Noise Using Wavelet Filters A) Daubechies, B) Bi-Orthogonal, C) Symlet, and D) Coiflet Filters.

Fig. 4 shows the denoised images by using wavelet filters. These filters were removed the most of the noise along with some image information as they considered as noise. Hence the PSNR values and SSIM values of the denoised image are low when compared to the noisy image.

\begin{tabular}{lllllll}
\multicolumn{7}{c}{ Table 1: Results Obtained by the Wavelet Filters } \\
\hline \multirow{2}{*}{ Wavelet Filter } & \multicolumn{2}{c}{ Salt \& Pepper } & Gaussian & \multicolumn{3}{c}{ Poisson } \\
& PSNR & SSIM & PSNR & SSIM & PSNR & SSIM \\
\hline Doubechies & 22.01 & 0.94 & 18.16 & 0.842 & 21.35 & 0.93 \\
Bi-orthogonal & 22.85 & 0.95 & 19.95 & 0.905 & 23.03 & 0.95 \\
Symlet & 21.97 & 0.94 & 18.24 & 0.847 & 21.41 & 0.93 \\
Coiflet & 22.08 & 0.942 & 18.25 & 0.840 & 21.43 & 0.93 \\
\hline
\end{tabular}

Table 1 shows the performance results obtained by the four different wavelet filters.

\subsection{Denoising using DTCWT}

(A)

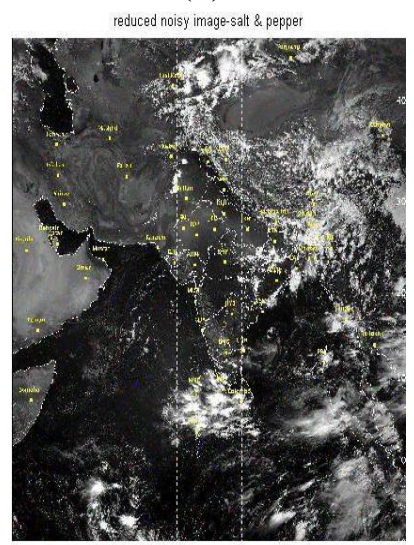

(B)

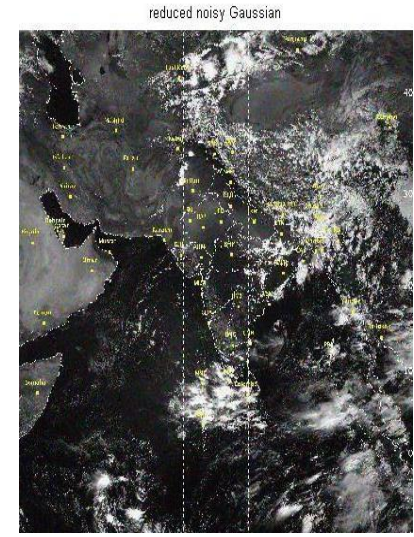

(C)

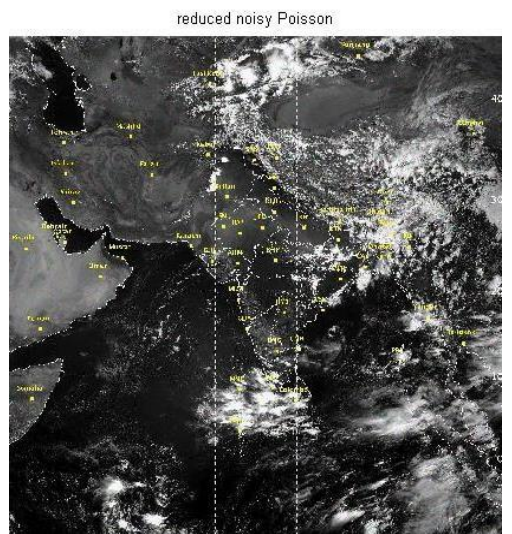

Fig. 5: Denoised Images by Using DTCWT A) Salt \& Pepper Noise, B). Gaussian Noise and C). Poisson Noise.

The Fig.5 shows the denoised Kalpana Satellite image using Dual Tree Complex Wavelet Transform for Salt \& Pepper noise, Gaussian noise and Poisson noise.

Table 2: Performance Results Obtained by Using DTCWT

\begin{tabular}{lllll}
\hline \multirow{2}{*}{ Type of Noise } & \multicolumn{2}{l}{ Noisy Image } & \multicolumn{2}{c}{ Denoised Image } \\
& PSNR & SSIM & PSNR & SSIM \\
\hline Gaussian & 31.48 & 0.968 & 42.23 & 0.991 \\
Salt \& Pepper & 35.33 & 0.978 & 47.92 & 0.993 \\
Poisson & 40.52 & 0.986 & 52.86 & 0.998 \\
\hline
\end{tabular}

Performance results of DTCWT based denoising method shown in Table 2 for both noisy and denoised Kalpana Satellite image.

\section{Conclusion}

This paper presents a noise reduction technique based on Dual Tree Complex Wavelet Transform. PSNR and SSIM values are calculated for different noisy and denoised images. The DTCWT based technique shows the better results compared to basic wavelet filters. So the image denoised method based on DTCWT removes the noise from an image and it is also well suitable for Satellite images. During pre-processing of satellite data, it is better to use DTCWT method instead of spatial filters and basic wavelets for better analysis of satellite data.

\section{References}

[1] M. Fierro, Ho-Gun Ha, Yeong-Ho Ha, "Noise reduction based on partial-reference, dual-tree complex wavelet transform shrinkage", IEEE Transactions on Image Processing, Vol. 22, No. 5, pp. 1859 1872, MAY 2013.

[2] P. Giri Prasad, S.Varadarajan, "An algorithm for Estimation of Rainfall over Chennai using Spatial Gradient Method", International journal of Electronics Engineering, Vol. 10, Issue 1, pp. 130-135, Jan 2018- June 2018

[3] Sateesh Kumar Kanagala and Sreenivasulu.G, "Landsat 8: UDTCWT based Denoising and Estimation", International Conference on Communication and Signal Processing, April 3-5, 2018.

[4] N. G. Kingsbury, "Image Processing with complex wavelets," Philos. Trans. Math. Phys. Eng. Sci., vol. 357, no. 1760, pp. 2543-2560, 1999.

[5] I.W. Selesnick, R. G. Baraniuk, and N. G. Kingsbury, "The dual-tree complex wavelet transform: A coherent framework for multiscale signal and image Processing," vol. 22, no.6, pp. 123-151, Nov. 2005.

[6] M. Fierro, W.J. Kyung, and Y.-H. Ha, "Dual-tree complex wavelet transform based denoising for random spray image enahcement methods," in Proc. 6th Eur. Conf. Colour Graph., Imag. Vis., pp. 194-199, 2012.

[7] Naga Lingamaiah Kurva, S. Varadarajan, "Partial Reference Based Noise Reduction Using DTCWT", International Journal of Advance Engineering and Research Development, Vol.4, Issue. 7, July - 2017. 